\title{
The Factors Associated With Health-Related Quality of Life in Stroke Survivors Age 40 and Older
}

\author{
Na Eun Jeon, MD, Kyoung Min Kwon, MD, Yeo Hyung Kim, MD, Jung Soo Lee, MD \\ Department of Rehabilitation Medicine, Uijeongbu St. Mary's Hospital, College of Medicine, \\ The Catholic University of Korea, Uijeongbu, Korea
}

\begin{abstract}
Objective To evaluate the association between clinical and socio-demographic status and health-related quality of life (HR-QoL) in stroke survivors age 40 and older in the Korean population.

Methods The relationship between stroke and HR-QoL was investigated using data from the Korea National Health and Nutrition Examination Survey (KNHANES), conducted from 2007-2012. The total number of respondents was 24,050 and 757 stroke survivors were included. Specifically, we investigated factors associated with the lowering of HR-QoL in a group of Korean adult stroke patients age 40 and older after adjusting for sociodemographic factors, anthropometric measurements, and clinical comorbidities. Adjusted effects of covariates on EuroQol-visual analog scales (EQ-VAS) scores were estimated with a complex-samples general linear model and multivariate-adjusted odds ratios of the lowest quintile versus highest quintile of the EQ-VAS scores in the stroke group were estimated with a complex-samples logistic regression model.

Results Socio-demographic, anthropometric factors, and clinical comorbidities significantly differed between the stroke and non-stroke groups, except regarding gender and residential area. The EQ-VAS was significantly lower in the stroke than non-stroke group. Anxiety/depression problems significantly lowered EQ-VAS, as did low education level, smoking, mobility and usual activities problems. Also, aging in 1-year increments and cardiovascular disease had a significant effect on lower EQ-VAS in stroke survivors.

Conclusion People with a stroke have significantly lower HR-QoL than healthy subjects. Therefore, managing depression, smoking, or cardiovascular disease and maintenance of physical function may be priority goals in improving HR-QoL after a stroke.
\end{abstract}

Keywords Stroke, Quality of life, Visual analog scale

Received October 26, 2016; Accepted January 6, 2017

Corresponding author: Jung Soo Lee

Department of Rehabilitation Medicine, Uijeongbu St. Mary's Hospital, College of Medicine, The Catholic University of Korea, 271 Cheonbo-ro, Uijeongbu 11765, Korea. Tel: +82-31-820-3178, Fax: +82-31-820-3177, E-mail: drlee71@naver.com

ORCID: Na Eun Jeon (http://orcid.org/0000-0003-3239-8490); Kyoung Min Kwon (http://orcid.org/0000-0002-9021-7099); Yeo Hyung Kim (http:// orcid.org/0000-0002-3951-7205); Jung Soo Lee (http://orcid.org/0000-0002-3807-4377).

(c) This is an open-access article distributed under the terms of the Creative Commons Attribution Non-Commercial License (http://creativecommons.org/ licenses/by-nc/4.0) which permits unrestricted noncommercial use, distribution, and reproduction in any medium, provided the original work is properly cited. Copyright $\odot 2017$ by Korean Academy of Rehabilitation Medicine 


\section{INTRODUCTION}

Stroke is a leading cause of adult deaths and a major cause of acquired long-term disability in Korea as well as worldwide [1]. In the past several decades, the age-adjusted mortality rate of stroke has declined because of development in disease intervention and prevention. However, there are still approximately 105,000 Korean people suffering from new or recurrent strokes annually, and the prevalence is likely to continue increasing because of the rapidly growing elderly population $[2,3]$. Therefore, the significance of quality of life (QoL) in stroke survivors living longer with stroke sequelae and long-term disability has been emphasized. Concepts in stroke treatment have expanded from decreasing mortality and morbidity to improving the functional level and QoL in stroke survivors [4].

QoL has been defined by the World Health Organization (WHO) as 'individuals' perception of their position in life according to their purpose, expectations, standards, and concerns within the context of the culture and value system in which they live $[5,6]$. In a related concept, healthrelated quality of life (HR-QoL) is a construct that focuses on an individual's subjective perception of health status and consists of physical, mental, and social dimensions [7].

The national-level studies about this topic have not been conducted sufficiently in the Korean stroke survivor population, widely different from other countries regarding the genetic, socio-economic factors and cultural environments. Therefore, little is known about HR-QoL for Korean patients after strokes. Rehabilitation should play a major role in reducing disability and enhancing HRQoL in stroke survivors. It is critical to evaluate patients' perception of health and their individual concept of disability $[8,9]$.

In this study, we investigated the impact of stroke on HR-QoL in Korean adults age 40 and older and the factors associated with HR-QoL in stroke survivors.

\section{MATERIALS AND METHODS}

\section{Data collection and subjects}

This study was based on data obtained by the Korea National Health and Nutrition Examination Survey (KNHANES), conducted from 2007-2012, which has been conducted periodically since 1998 to assess the health and nutritional status of the civilian non-institutionalized population in Korea. It is a cross-sectional and nationally representative survey, conducted by the Division of Chronic Disease Surveillance, Korea Centers for Disease Control and Prevention (KCDC). The survey used a stratified, multistage probability-sampling design to select respondents within household units. It consisted of a health examination, nutrition survey, and health and behavior questionnaire that included HR-QoL aspects. The survey collected data via household interviews and by direct standardized physical examinations conducted in specially equipped mobile examination centers.

The Institutional Review Board of the KCDC approved the study protocol (IRB no. UC16EISI0085). Participants provided written informed consent before survey administration. Among those participating in the survey between January 2007-December 2012, our study included adults age 40 and older answering 'Yes' when asked if they had stroke symptoms in the self-reported health and behavior questionnaire. For the KNHAES (2007-2012), the number of respondents age 40 and older was 24,050 and a total of 757 stroke survivors were included in this study.

\section{Dependent variable}

Measures of HR-QoL: EuroQol-visual analog scale (EQVAS)

HR-QoL was evaluated using the EQ-VAS from the EQ5D questionnaire, developed by the International European Quality of Life Group, to be applied internationally and standardized as a measure of QoL. EQ-VAS is a scale numbered from 0 to 100 ( 0 is the worst and 100 is the best health status) that allows individuals to place themselves according to how they perceive their overall health status. Individual respondents were shown a demonstration of this scale, received verbal explanation, and were asked to mark an $\mathrm{X}$ on the scale and to write the number marked on the scale in the box below to indicate how good or bad their health was at that time.

\section{Independent variable}

Socio-demographic factors \& health problems

Socio-demographic factors evaluated included age, gender, marital status, education level, residential area, household annual income, occupation, and smoking 
status. Subjects were considered urban residents if their address was in Seoul, Busan, Daegu, Incheon, Gwangju, Daejeon, or Ulsan. Education level was classified into four categories: elementary school, middle school, high school, and college or university degree or higher. Household annual income was divided into national quartiles, from Q1 (lowest) to Q4 (highest). Occupation and smoking status were dichotomized as having a current occupation or not and current smokers and nonsmokers, including ex-smokers. Five dimensions of EQ5D (mobility, self-care, usual activities, pain/discomfort, anxiety/depression), indicating how the subject's health was today, were dichotomized as having problems versus not having problems. Duration after a stroke was divided into ' 5 years or more' and 'less than 5 years'.

\section{Anthropometric measurements and clinical comorbidities}

Body mass index (BMI, $\mathrm{kg} / \mathrm{m}^{2}$ ), waist circumference $(\mathrm{cm})$, and arterial blood pressure were estimated during the health examination survey by trained medical staff following a standardized procedure. Fasting blood

Table 1. Socio-demographic and clinical characteristics of study participants

\begin{tabular}{|c|c|c|c|}
\hline Characteristic & No stroke $(n=23,293)$ & Stroke $(n=757)$ & p-value \\
\hline Age (yr) & $55.40 \pm 0.13$ & $66.33 \pm 0.52$ & $<0.001^{*}$ \\
\hline Gender, male (\%) & $47.78 \pm 0.30$ & $52.05 \pm 2.30$ & 0.07 \\
\hline Marital status, married (\%) & $83.38 \pm 0.35$ & $69.45 \pm 2.09$ & $<0.001^{*}$ \\
\hline Education level (\%) & & & $<0.001^{*}$ \\
\hline Elementary school & $32.16 \pm 0.55$ & $58.48 \pm 2.35$ & \\
\hline Middle school & $15.75 \pm 0.33$ & $16.07 \pm 1.84$ & \\
\hline High school & $31.80 \pm 0.47$ & $17.26 \pm 1.75$ & \\
\hline College or University & $20.29 \pm 0.55$ & $8.19 \pm 1.26$ & \\
\hline Residential area, rural (\%) & $54.70 \pm 0.80$ & $58.60 \pm 2.50$ & 0.12 \\
\hline Household income (\%) & & & $<0.001^{*}$ \\
\hline Low & $20.88 \pm 0.49$ & $45.20 \pm 2.28$ & \\
\hline Low-middle & $26.06 \pm 0.51$ & $25.43 \pm 1.95$ & \\
\hline Middle-high & $25.43 \pm 0.47$ & $15.62 \pm 1.67$ & \\
\hline High & $27.63 \pm 0.64$ & $13.75 \pm 1.66$ & \\
\hline Occupation, employed (\%) & $63.43 \pm 0.49$ & $31.03 \pm 2.24$ & $<0.001^{*}$ \\
\hline Current smoker (\%) & $22.49 \pm 0.36$ & $17.73 \pm 1.82$ & $0.02 *$ \\
\hline Diabetes mellitus (\%) & $35.68 \pm 0.46$ & $49.57 \pm 2.38$ & $<0.001^{*}$ \\
\hline Hypertension (\%) & $64.88 \pm 0.49$ & $87.71 \pm 1.41$ & $<0.001^{*}$ \\
\hline Arthritis (\%) & $20.11 \pm 0.34$ & $30.18 \pm 2.06$ & $<0.001^{*}$ \\
\hline Angina or MI (\%) & $3.03 \pm 0.13$ & $9.70 \pm 1.28$ & $<0.001^{*}$ \\
\hline Heath problems (\%) & & & $<0.001^{*}$ \\
\hline Mobility & $19.18 \pm 0.35$ & $54.46 \pm 2.32$ & \\
\hline Self-care & $5.18 \pm 0.18$ & $25.85 \pm 1.98$ & \\
\hline Usual activities & $12.10 \pm 0.29$ & $43.60 \pm 2.28$ & \\
\hline Pain/discomfort & $27.42 \pm 0.42$ & $52.06 \pm 2.27$ & \\
\hline Anxiety/depression & $13.29 \pm 0.31$ & $27.07 \pm 1.79$ & \\
\hline EQ-VAS & $73.08 \pm 0.17$ & $60.69 \pm 1.07$ & $<0.001^{*}$ \\
\hline Body mass index $\left(\mathrm{kg} / \mathrm{m}^{2}\right)$ & $24.04 \pm 0.03$ & $24.20 \pm 0.12$ & $<0.001^{*}$ \\
\hline Waist circumference $(\mathrm{cm})$ & $82.91 \pm 0.10$ & $85.41 \pm 0.38$ & $<0.001^{*}$ \\
\hline
\end{tabular}

Values are presented as mean \pm standard error.

MI, myocardial infarction; EQ-VAS, EuroQol-visual analog scale.

${ }^{*} \mathrm{p}<0.05$. 
Table 2. Multivariate linear regression analyses of EQ-VAS in the stroke group

\begin{tabular}{|c|c|c|c|}
\hline & Beta & $95 \% \mathrm{CI}$ & p-value \\
\hline Age (yr) (by 1-yr increment) & $-0.16 \pm 0.11$ & -0.37 to 0.06 & 0.15 \\
\hline Body mass index (by 1 increment) & $0.67 \pm 0.54$ & -0.40 to 1.72 & 0.22 \\
\hline Waist circumference (cm) (by $1 \mathrm{~cm}$ increment) & $-0.32 \pm 0.18$ & -0.68 to 0.43 & 0.84 \\
\hline \multicolumn{4}{|l|}{ Gender } \\
\hline Male & $1.51 \pm 2.27$ & -2.95 to 5.98 & 0.51 \\
\hline Female (ref) & 0 & & \\
\hline \multicolumn{4}{|l|}{ Marital status } \\
\hline Married & $0.29 \pm 2.29$ & -4.22 to 4.79 & 0.90 \\
\hline Single, divorced, widow or widower (ref) & 0 & & \\
\hline \multicolumn{4}{|l|}{ Education level } \\
\hline Elementary school & $-8.66 \pm 3.68$ & -15.90 to -1.43 & $0.02 *$ \\
\hline Middle school & $-5.61 \pm 3.12$ & -11.75 to 0.54 & 0.07 \\
\hline High school & $-7.98 \pm 3.51$ & -14.89 to -1.08 & $0.02^{*}$ \\
\hline College or university (ref) & 0 & & \\
\hline \multicolumn{4}{|l|}{ Residential area } \\
\hline Rural & $2.31 \pm 1.85$ & -1.32 to 5.95 & 0.21 \\
\hline Urban (ref) & 0 & & \\
\hline \multicolumn{4}{|l|}{ Household income } \\
\hline Low & $-2.38 \pm 3.03$ & -8.34 to 3.59 & 0.43 \\
\hline Low-middle & $0.60 \pm 3.03$ & -5.37 to 6.56 & 0.84 \\
\hline Middle-high & $0.43 \pm 3.22$ & -5.91 to 6.77 & 0.90 \\
\hline High (ref) & 0 & & \\
\hline \multicolumn{4}{|l|}{ Occupation, employed } \\
\hline Yes & $2.43 \pm 2.20$ & -1.90 to 6.76 & 0.27 \\
\hline No (ref) & 0 & & \\
\hline \multicolumn{4}{|l|}{ Current smoker } \\
\hline No & $5.03 \pm 2.47$ & 0.18 to 9.89 & $0.04^{*}$ \\
\hline Yes (ref) & 0 & & \\
\hline \multicolumn{4}{|l|}{ Mobility problem } \\
\hline No & $5.06 \pm 2.50$ & 0.15 to 9.96 & $0.04^{*}$ \\
\hline Yes (ref) & 0 & & \\
\hline \multicolumn{4}{|l|}{ Self-care problem } \\
\hline No & $1.50 \pm 2.67$ & -3.74 to 6.74 & 0.57 \\
\hline Yes (ref) & 0 & & \\
\hline \multicolumn{4}{|l|}{ Usual activities problem } \\
\hline No & $6.49 \pm 2.79$ & 1.01 to 11.96 & $0.02^{*}$ \\
\hline Yes (ref) & 0 & & \\
\hline \multicolumn{4}{|l|}{ Pain/discomfort problem } \\
\hline No & $2.94 \pm 2.38$ & -1.74 to 7.62 & 0.22 \\
\hline Yes (ref) & 0 & & \\
\hline \multicolumn{4}{|l|}{ Anxiety/depression problem } \\
\hline No & $8.86 \pm 2.03$ & 4.88 to 12.85 & $<0.001^{*}$ \\
\hline Yes (ref) & 0 & & \\
\hline
\end{tabular}


Table 2. Continued

\begin{tabular}{|lccc|}
\hline & Beta & $\mathbf{9 5 \%}$ CI & p-value \\
\hline Diabetes mellitus & & -3.24 to 4.10 & 0.82 \\
\hline No & $0.43 \pm 1.87$ & & \\
\hline Yes (ref) & 0 & -3.58 to 6.14 & 0.52 \\
\hline Hypertension & $1.23 \pm 2.47$ & & 0.78 \\
\hline No & 0 & -3.78 to 5.04 & 0.21 \\
\hline Yes (ref) & & & \\
\hline Arthritis & $0.63 \pm 2.24$ & -8.58 to 1.91 & \\
\hline No & 0 & & 0.35 \\
\hline Yes (ref) & $-3.34 \pm 2.67$ & & \\
\hline Angina or MI & 0 & -0.37 to 0.06 & \\
\hline No & & & \\
\hline Yes (ref) & $-0.16 \pm 0.11$ & 0 & \\
\hline Duration of stroke (yr) & & & \\
\hline$<5$ & & & \\
\hline$\geq 5$ &
\end{tabular}

Values are presented as mean \pm standard error.

EQ-VAS, EuroQol-visual analog scale; CI, confidence interval; MI, myocardial infarction; ref, reference. ${ }^{*} \mathrm{p}<0.05$.

glucose level was measured by an enzymatic method. Diabetes mellitus was defined as a serum fasting glucose level $\geq 126 \mathrm{mg} / \mathrm{dL}$, use of anti-diabetic medications, or a physician diagnosis of diabetes mellitus, and impaired fasting glucose was defined as a fasting plasma glucose level of $100-125 \mathrm{mg} / \mathrm{dL}$, that were dichotomized as having diabetes mellitus. Hypertension was defined as systolic blood pressure $\geq 140 \mathrm{mmHg}$, diastolic blood pressure $\geq 90 \mathrm{mmHg}$, or use of anti-hypertensive medications. Arthritis and coronary heart disease (myocardial infarction [MI] or angina pectoris) were defined according to if subjects suffered from pain or the disease at that time of self-reporting.

\section{Statistical analysis}

Statistical analyses were conducted using the SAS ver. 9.4 software (SAS Institute Inc., Cary, NC, USA). The pvalues $<0.05$ were considered statistically significant. Data are presented as estimated mean $\% \pm$ standard error (with $95 \%$ confidence intervals [CI]) for categorical variables and estimated mean \pm standard error $(95 \% \mathrm{CI})$ for continuous variables. Categorical variables and continuous variables were analyzed by complex-samples descriptive and tabulated methods, respectively. Adjusted effects of covariates on EQ-VAS score were estimated using a complex-samples general linear model (CSGLM) and the multivariate-adjusted odds ratios of the lowest quintile versus the highest quintile of the EQ-VAS scores in the stroke group were estimated using a complex-samples logistic regression model (CSLRM).

\section{RESULTS}

Of 24,050 respondents age 40 and older, 757 reported that they had suffered a stroke. The socio-demographic and anthropometric characteristics and clinical comorbidities of the 24,050 respondents are shown in Table 1 . The mean age was $66.33 \pm 0.52$ years (95\% CI, 65.30-67.36) and the percentage of males was $52.05 \% \pm 2.30 \%$ (95\% CI, 47.53-56.54) in the stroke group ( $\mathrm{n}=757)$. The EQ-VAS was significantly lower in the stroke $(60.69 \pm 1.07$; $95 \% \mathrm{CI}$, 58.59-62.79) than non-stroke group (73.08 $\pm 0.17 ; 95 \% \mathrm{CI}$, 72.75-73.41) $(\mathrm{p}<0.001)$. Socio-demographic and anthropometric factors and clinical comorbidities significantly differed between the groups, except regarding gender and residential area $(\mathrm{p}<0.05)$.

Multivariate linear regression analyses of EQ-VAS in the stroke group are summarized in Table 2. The factors that decreased the EQ-VAS in the stroke group were low education level, smoking, mobility problem, usual activities 
Table 3. Multivariate-adjusted ORs of the lowest quintile of the EQ-VAS for the highest quintile in the stroke group

\begin{tabular}{|c|c|c|}
\hline & Adjusted OR & p-value \\
\hline Age (yr) (by 1 -yr increment) & $1.03(1.00-1.06)$ & $0.04^{*}$ \\
\hline $\begin{array}{l}\text { Body mass index } \\
\text { (by } 1 \text { increment) }\end{array}$ & $0.98(0.85-1.13)$ & 0.79 \\
\hline $\begin{array}{l}\text { Waist circumference }(\mathrm{cm}) \\
\text { (by } 1 \mathrm{~cm} \text { increment) }\end{array}$ & $1.01(0.96-1.06)$ & 0.65 \\
\hline \multicolumn{3}{|l|}{ Gender } \\
\hline Male & $0.63(0.34-1.18)$ & 0.15 \\
\hline Female (ref) & 1.00 & \\
\hline \multicolumn{3}{|l|}{ Marital status } \\
\hline Married & $0.97(0.51-1.84)$ & 0.93 \\
\hline $\begin{array}{l}\text { Single, divorced, widow or } \\
\text { widower (ref) }\end{array}$ & 1.00 & \\
\hline \multicolumn{3}{|l|}{ Education level } \\
\hline Elementary school & $2.03(0.59-6.99)$ & 0.26 \\
\hline Middle school & $1.26(0.35-4.52)$ & 0.72 \\
\hline High school & $2.27(0.61-8.47)$ & 0.22 \\
\hline College or university (ref) & 1.00 & \\
\hline \multicolumn{3}{|l|}{ Residential area } \\
\hline Rural & $0.72(0.45-1.15)$ & 0.17 \\
\hline Urban (ref) & 1.00 & \\
\hline \multicolumn{3}{|l|}{ Household income } \\
\hline Low & $2.23(0.98-5.08)$ & 0.06 \\
\hline Low-middle & $1.09(0.46-2.56)$ & 0.85 \\
\hline Middle-high & $1.35(0.49-3.77)$ & 0.56 \\
\hline High (ref) & 1.00 & \\
\hline \multicolumn{3}{|l|}{ Occupation, employed } \\
\hline Yes & $0.67(0.37-1.23)$ & 0.20 \\
\hline No (ref) & 1.00 & \\
\hline \multicolumn{3}{|l|}{ Current smoker } \\
\hline Yes & $0.44(0.23-0.85)$ & $0.02^{*}$ \\
\hline No (ref) & 1.00 & \\
\hline \multicolumn{3}{|l|}{ Mobility problem } \\
\hline No & $0.54(0.29-1.01)$ & 0.05 \\
\hline Yes (ref) & 1.00 & \\
\hline \multicolumn{3}{|l|}{ Self-care problem } \\
\hline No & $1.32(0.67-2.59)$ & 0.42 \\
\hline Yes (ref) & 1.00 & \\
\hline \multicolumn{3}{|l|}{ Usual activities problem } \\
\hline Yes & $0.52(0.27-1.02)$ & 0.06 \\
\hline No (ref) & 1.00 & \\
\hline \multicolumn{3}{|l|}{ Pain/discomfort problem } \\
\hline No & $0.65(0.36-1.15)$ & 0.14 \\
\hline Yes (ref) & 1.00 & \\
\hline
\end{tabular}

Table 3. Continued

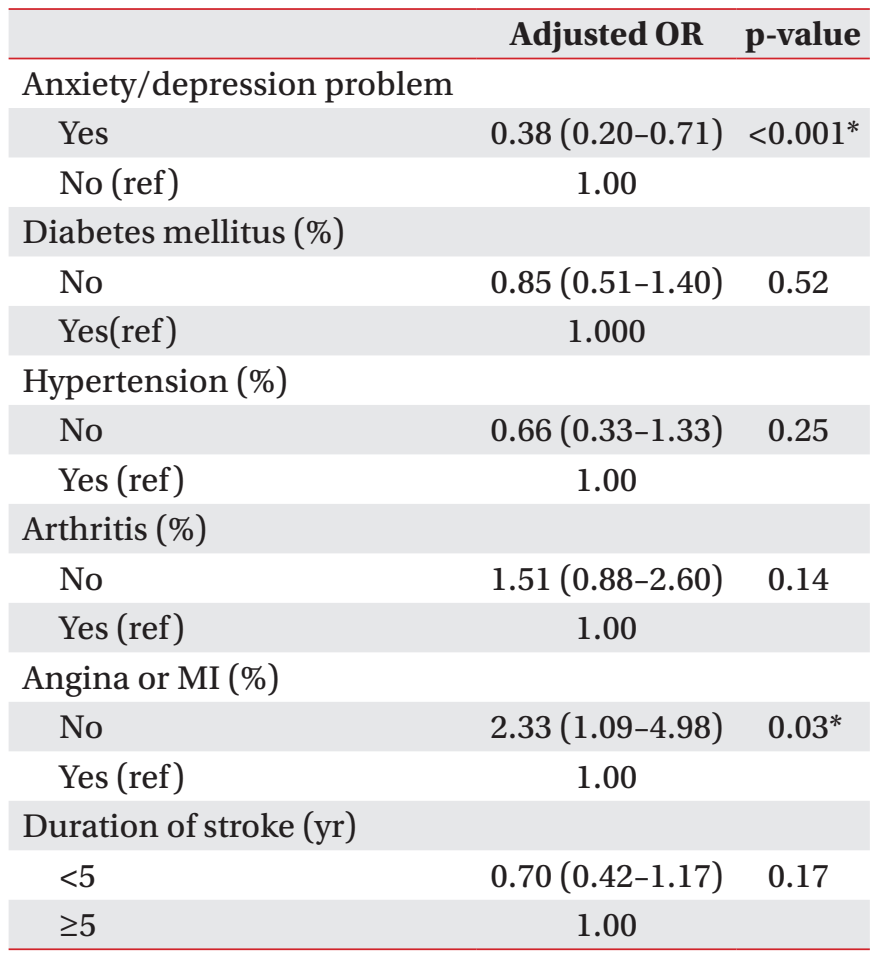

OR, odds ratio; EQ-VAS, EuroQol-visual analog scale; CI, confidence interval; MI, myocardial infarction; ref, reference.

${ }^{*} \mathrm{p}<0.05$.

problems, and anxiety/depression problems after adjusting covariates using the CSGLM. The EQ-VAS scores were estimated as $-8.66 \pm 3.68$ (95\% CI, -15.90 to -1.43$)$ lower in elementary school graduates and $-7.98 \pm 3.51$ (95\% CI, -14.89 to -1.08) lower in high school graduates than college graduates $(\mathrm{p}<0.05)$. The EQ VAS score of non-smokers was estimated as 5.03 \pm 2.47 (95\% CI, 0.18-9.89) higher than smokers. The EQ VAS scores of respondents without mobility (5.06 \pm 2.50 ; 95\% CI, 0.15-9.96), usual activities (6.49 $\pm 2.79 ; 95 \%$ CI, 1.01-11.96) and anxiety/depression (8.86 $\pm 2.03,95 \%$ CI, 4.88-12.85) problems were estimated as higher than those with the problems $(\mathrm{p}<0.05)$. This also revealed, surprisingly, that the duration of stroke and the medical comorbidities, such as diabetes, hypertension, arthritis and cardiovascular diseases, did not lower EQ VAS in stroke survivors ( $\mathrm{p}>0.05)$.

Multivariate-adjusted odds ratios (ORs) of the lowest quintile of the EQ-VAS score for the highest quintile in stroke group are shown in Table 3 . A subgroup analysis for respondents in the stroke group was conducted to examine the impact of covariates on lowering EQ-VAS. The 
EQ-VAS scores were divided into quintiles in the stroke group, and ORs of the lowest quintile of EQ-VAS scores were compared to the highest quintile in stroke group after adjusting for age, BMI, waist circumference, sex, marital status, education level, residential area, household income, employed status, smoking, mobility, self-care, usual activities, pain/discomfort, anxiety/depression, duration of stoke and medical comorbidities (diabetes mellitus, hypertension, arthritis, angina/MI) using a logistic regression procedure. The results indicated that aging by a one-year increment ( $\mathrm{OR}=1.03 ; 95 \% \mathrm{CI}, 1.00-1.06$ ), smoking ( $\mathrm{OR}=0.44 ; 95 \% \mathrm{CI}, 0.23-0.85$ ), anxiety/depression problems (OR=0.38; 95\% CI, 0.20-0.71) and angina/ MI (OR=2.33; 95\% CI, 1.09-4.98) had a significant effect on lower EQ-VAS. However, duration after stroke and other medical comorbidities, except angina/MI, known risk factors in chronic diseases that hinder daily activities, did not have a significant effect on lower EQ-VAS $(p>0.05)$.

\section{DISCUSSION}

Due to the increasing significance of HR-QoL as an indicator of stroke outcomes, several utility measures have been introduced to assess patient-reported health status, including the EQ-5D, a standardized, simple, and generic measure of health perception consisting of a descriptive system with five dimensions and an EQ-VAS. Validation of EQ-5D in Korean populations has been demonstrated in other studies [10]. Previous studies have revealed that several factors including age, sex, functional status, socioeconomic status, psychomotor impairment, and medical comorbidities influence HR-QoL in stroke survivors [11-13]. However, there is no consensus on the factors affecting HR-QoL. Studies about HR-QoL of stroke patients in Korean populations are also insufficient, of greater significance considering that genetic factors, socio-economic, and cultural environments differ among countries. From previous studies, we established the hypothesis that HR-QoL in stroke survivors is influenced by a complex interplay between the stroke, clinical risk factors or comorbidities, and socio-economic status.

This study, using representative information based on a large population-based, cross-sectional, nationally representative survey, demonstrated that the EQ-VAS score in the stroke group age 40 and older was signifi- cantly decreased compared to the non-stroke group in Korean adults. Results revealed that factors lowering HR-QoL in the stroke group included education level, smoking, mobility, usual activities, anxiety/depression and cardiovascular disease, such as angina or MI, but not gender, marriage, residential area, income, occupation, BMI, self-care degree, pain problems and other medical comorbidities (diabetes, hypertension or arthritis), after adjusting for covariates. Previous studies have indicated that low socio-economic status, such as low education level and low income, is associated with low HR-QoL $[14,15]$. Low socio-economic status may place individuals at risk with poorer health for various reasons, such as less access to healthcare, poorer living conditions, less knowledge about the negative consequences of healthcompromising behavior, and greater psychological stress. This has been reported in association with stroke and for other chronic diseases, such as cancer and coronary heart disease [16]. Depression is a known modifiable factor that has been associated with low HR-QoL in stroke survivors in many studies [17-19]. Post-stroke depression is a common psychological complication in stroke survivors and is a barrier that prevents them from recovering from neurological deficits and improving functional outcomes. The influence of age or sex on HR-QoL in stroke survivors has remained inconclusive. Some studies reported that female stroke survivors have lower HR-QoL and functional recovery compared with males [20-23]. Conversely, Jun et al. [15] said male stroke survivors revealed poor HR-QoL than females. In our study, aging by a 1-year increment had a significant effect on lower EQVAS. Mobility and usual activity domains are key factors in performing independent functions and play critical roles in determining the quality of life after strokes. Our study revealed that mobility or usual activities problem were associated with lower HR-QoL of stroke survivors. Previous studies also had similar results that stroke patients with limitations in mobility and usual activities were the most likely to have the lowest scores of HR-QoL [24]. Cardiovascular disease known to be major cause of stroke, such as Angina or MI, was associated with the lowest quintile of EQ-VAS score in stroke patients. However, other medical comorbidities, except cardiovascular disease, were not significantly associated with lower HRQoL in the stroke group. Therefore, in stroke survivors managing cardiovascular disease is expected to prevent 
recurrent stroke and lower HR-QoL after a stroke. In this study, current smoking status is a major modifiable factor associated with low HR-QOL of stroke patients. It is consistent with results of several previous studies [2529]. Interestingly, in the population without strokes, the self-rated subjective health score of current smokers was rather higher than the non-smokers group [30]. There were two reasons to explain these contradictory results. First, smoking affected so many factors that may act as potentially confounding variables. Another reason is that unless smoking directly damages their health, relatively healthy smokers may not believe that smoking endangers their health state despite public health warnings. However, if they suffer from a major disease such as a stroke, disease survivors would agree smoking is detrimental to one's health, and they think that their health state is worse than non-smokers or former smokers that successfully quit smoking. This fact indicates that many stroke survivors probably want to stop smoking, but there are barriers to smoking cessation. In other studies, environmental factors such as family smokers and barriers of addiction and boredom played a central role in persistent smoking [31]. Therefore, a medical team and health ministry should more actively provide smoking cessation programs to stroke survivors and support public funding if necessary. Additionally, stroke survivors and their caregivers should receive counseling for smoking cessation.

The important thing in managing HR-QoL of stroke survivors is to control the modifiable factors, i.e., smoking state, depressive mood, mobility or usual activities problems, and cardiovascular disease. Therefore, rehabilitation for stroke patients should be enforced in a direction that may compensate for these modifiable factors and minimize disability. In addition, a welfare policy for aged stroke patients or stroke survivors with low socioeconomic class to receive proper rehabilitation treatment is needed.

To our knowledge, this is the first study that investigated the association between widely varied variables and HR-QoL of stroke survivors using KNHANES, which was large and powerful data set in Korea and conducted by the KCDC that has public confidence and a wellstructured method. The examined variables considered socio-economic, welfare and medical aspects together. Our study was based on representative information collected in a nationwide population with a high response rate, which also had several limitations. It was a crosssectional survey and causal relationships could not be determined. We did not assess all medical comorbidities or functional status that may affect HR-QoL of strokes, because it was not a survey only about strokes. However, this study examined several comorbidity covariates that are risk factors for cerebrovascular diseases or hinder daily activities.

Results revealed that most of medical comorbidities, except cardiovascular diseases in CSLRM, had no statistically significant association with lowering HR-QoL of stroke survivors in Korean adults. This indicates that clinicians should not overlook emotional factors such as anxiety or depression, and daily usual activity function. Indeed, stroke patients may need more functional and emotional support beyond treatment of their chronic disease.

In conclusion, people that have had a stroke have significantly lower HR-QoL than healthy subjects. Therefore, managing depression or anxiety, smoking and cardiovascular problems and maintenance of physical function may be a priority goal for improving the HR-QoL of stroke survivors in Korean adults.

\section{CONFLICT OF INTEREST}

No potential conflict of interest relevant to this article was reported.

\section{REFERENCES}

1. Mozaffarian D, Benjamin EJ, Go AS, Arnett DK, Blaha MJ, Cushman M, et al. Heart disease and stroke statistics-2016 update: a report from the American Heart Association. Circulation 2016;133:e38-360.

2. Lee HT, Shin J, Lim YH, Kim KS, Kim SG, Kim JH, et al. Health-related quality of life in coronary heart disease in Korea: the Korea National Health and Nutrition Examination Survey 2007 to 2011. Angiology 2015;66:326-32.

3. Hong KS, Bang OY, Kang DW, Yu KH, Bae HJ, Lee JS, et al. Stroke statistics in Korea. Part I. Epidemiology and risk factors: a report from the Korean stroke society and clinical research center for stroke. J Stroke 2013;15:2-20.

4. Chang WH, Sohn MK, Lee J, Kim DY, Lee SG, Shin YI, 
et al. Predictors of functional level and quality of life at 6 months after a first-ever stroke: the KOSCO study. J Neurol 2016;263:1166-77.

5. The World Health Organization Quality of Life Assessment (WHOQOL): development and general psychometric properties. Soc Sci Med 1998;46:1569-85.

6. Carod-Artal FJ, Egido JA. Quality of life after stroke: the importance of a good recovery. Cerebrovasc Dis 2009;27 Suppl 1:204-14.

7. Katona M, Schmidt R, Schupp W, Graessel E. Predictors of health-related quality of life in stroke patients after neurological inpatient rehabilitation: a prospective study. Health Qual Life Outcomes 2015;13:58.

8. Howitt SC, Jones MP, Jusabani A, Gray WK, Aris E, Mugusi F, et al. A cross-sectional study of quality of life in incident stroke survivors in rural northern Tanzania. J Neurol 2011;258:1422-30.

9. Donkor ES, Owolabi MO, Bampoh PO, Amoo PK, Aspelund T, Gudnason V. Profile and health-related quality of life of Ghanaian stroke survivors. Clin Interv Aging 2014;9:1701-8.

10. Kim SH, Ahn J, Ock M, Shin S, Park J, Luo N, et al. The EQ-5D-5L valuation study in Korea. Qual Life Res 2016;25:1845-52.

11. Haghgoo HA, Pazuki ES, Hosseini AS, Rassafiani M. Depression, activities of daily living and quality of life in patients with stroke. J Neurol Sci 2013;328:87-91.

12. Guajardo VD, Terroni L, Sobreiro Mde F, Zerbini MI, Tinone G, Scaff M, et al. The influence of depressive symptoms on quality of life after stroke: a prospective study. J Stroke Cerebrovasc Dis 2015;24:201-9.

13. Franceschini M, La Porta F, Agosti M, Massucci M; ICR2 group. Is health-related-quality of life of stroke patients influenced by neurological impairments at one year after stroke? Eur J Phys Rehabil Med 2010;46:389-99.

14. Lee K, Lim HT, Hwang SS, Chae DW, Park SM. Socioeconomic disparities in behavioural risk factors for cancer and use of cancer screening services in Korean adults aged 30 years and older: the Third Korean National Health and Nutrition Examination Survey, 2005 (KNHANES III). Public Health 2010;124:698-704.

15. Jun HJ, Kim KJ, Chun IA, Moon OK. The relationship between stroke patients' socio-economic conditions and their quality of life: the 2010 Korean community health survey. J Phys Ther Sci 2015;27:781-4.
16. Alter DA, Iron K, Austin PC, Naylor CD; SESAMI Study Group. Socioeconomic status, service patterns, and perceptions of care among survivors of acute myocardial infarction in Canada. JAMA 2004;291:1100-7.

17. Hackett ML, Yapa C, Parag V, Anderson CS. Frequency of depression after stroke: a systematic review of observational studies. Stroke 2005;36:1330-40.

18. Klit H, Finnerup NB, Overvad K, Andersen G, Jensen TS. Pain following stroke: a population-based followup study. PLoS One 2011;6:e27607.

19. Pan JH, Song XY, Lee SY, Kwok T. Longitudinal analysis of quality of life for stroke survivors using latent curve models. Stroke 2008;39:2795-802.

20. Carod-Artal FJ, Gonzalez-Gutierrez JL, Herrero JA, Horan T, De Seijas EV. Functional recovery and instrumental activities of daily living: follow-up 1-year after treatment in a stroke unit. Brain Inj 2002;16:207-16.

21. Patel MD, McKevitt C, Lawrence E, Rudd AG, Wolfe CD. Clinical determinants of long-term quality of life after stroke. Age Ageing 2007;36:316-22.

22. Kwok T, Lo RS, Wong E, Wai-Kwong T, Mok V, Kai-Sing W. Quality of life of stroke survivors: a 1-year followup study. Arch Phys Med Rehabil 2006;87:1177-82.

23. Bushnell CD, Reeves MJ, Zhao X, Pan W, Prvu-Bettger J, Zimmer L, et al. Sex differences in quality of life after ischemic stroke. Neurology 2014;82:922-31.

24. Sprigg N, Selby J, Fox L, Berge E, Whynes D, Bath PM, et al. Very low quality of life after acute stroke: data from the Efficacy of Nitric Oxide in Stroke trial. Stroke 2013;44:3458-62.

25. Hong JW, Ku CR, Noh JH, Ko KS, Rhee BD, Kim DJ. Association between self-reported smoking and hemoglobin Alc in a Korean population without diabetes: the 2011-2012 Korean National Health and Nutrition Examination Survey. PLoS One 2015;10:e0126746.

26. Luger TM, Suls J, Vander Weg MW. How robust is the association between smoking and depression in adults? A meta-analysis using linear mixed-effects models. Addict Behav 2014;39:1418-29.

27. Jung SJ, Shin A, Kang D. Active smoking and exposure to secondhand smoke and their relationship to depressive symptoms in the Korea national health and nutrition examination survey (KNHANES). BMC Public Health 2015;15:1053.

28. Hong JY, Han K, Shin DH, Chun EM. Quality of life analysis and smoking correlation in symptomatic 
spine osteoarthritis: a nationwide health survey analysis of an elderly population with EQ-5D. PLoS One 2016;11:e0151315.

29. Bach JP, Riedel O, Pieper L, Klotsche J, Dodel R, Wittchen HU. Health-related quality of life in patients with a history of myocardial infarction and stroke. Cerebrovasc Dis 2011;31:68-76.

30. Park S, Ahn J, Lee BK. Self-rated subjective health status is strongly associated with sociodemographic factors, lifestyle, nutrient intakes, and biochemical indices, but not smoking status: KNHANES 2007-2012. J Korean Med Sci 2015;30:1279-87.

31. Lennon OC, Doody C, Ni Choisdealbh C, Blake C. Barriers to healthy-lifestyle participation in stroke: consumer participation in secondary prevention design. Int J Rehabil Res 2013;36:354-61. 